\title{
Quelques outils pédagogiques pour la classe de français de Spécialité
}

\section{Bahneanu Vitalina}

Zaporojan Ina

Zbant Ludmila

Université d'Etat de Moldova, Chisinau, Moldova

\section{Doi:10.5901/jesr.2013.v3n7p178}

\section{Résume}

Cet article propose différentes suggestions d'exercices qui peuvent être utilisés lors les cours de français de spécialité (cas pratiques-français juridique). Les auteurs partagent les méthodes connues pour introduire des activités dynamisantes de la classe du français juridique. Les récentes innovations concernant les approches participatives font de plus en plus de place aux méthodes visuelles (diagrammes, représentations visuelles) aux dépends des méthodes verbales (discussion de groupes, questions/réponses, simulation et jeu de rôle). Les «Technologies de I'Information et de la Communication pour I'Enseignement» (TICE) ont désormais leur place dans les curricula, qu'elles constituent une discipline autonome ou qu'elles se donnent pour une compétence transversale.

Mots clés: méthodes technique, contenu curriculaire, objectifs proposés, compétences linguistiques, simulation.

Cette étude est une de nature didactique. Son contenu récapitule les différentes méthodes qui président à l'enseignement du français langue étrangère à un public en voie de professionnalisation. Les réflexions présentées ont une allure générale, du moment que chaque méthode est choisie selon les priorités du contenu curriculaire et les objectifs proposés.

A nos yeux, il n y a pas de méthodes meilleures que d'autres, toute est question de priorité. Notre intention est une très simple: d'apporter aux personnes intéressées un éclairage sur les différents choix possibles. Cependant, le choix d'une méthode (ou d'une technique) ne dépend pas seulement de la prise en compte des principes d'apprentissage. II dépend aussi du contenu du programme, des conditions et contraintes que la méthode (ou technique) impose au professeur.

Il est donc important de pouvoir identifier les principales méthodes et techniques, d'évaluer leurs

avantages et leurs inconvénients pour effectuer le meilleur choix compte tenu des objectifs, des

circonstances et des contraintes. L'enseignant choisit la méthode qu'il va utiliser en fonction des buts qu'il veut atteindre, les personnes à former et le contexte. Le choix de la technique pédagogique tient compte des objectifs pédagogiques, des contraintes matérielles, de la forme et de la nature du contenu à enseigner ou des apprentissages à réaliser mais également de l'état du groupe en formation et de ses attentes. Pour utiliser une méthode il ne faut pas négliger quelques conditions de contrôle :

1. Le climat régnant au cours de FOU est-il agréable et motivant pour l'utilisation de la méthode respective?

2. Vos méthodes d'enseignements permettent-elles aux participants de faire connaître ou d'utiliser leurs anciennes connaissances?

3. La méthode permet-elle aux participants de mesurer leurs propres progrès?

La simulation reste au centre d'un cours de français de spécialité. F. Yaiche définit la simulation comme de la façon suivante » Prenez un lieu, de préférence clos : une ile, un immeuble, un village, un hôtel, un paquebot etc. Faitesle investir et décrire par des élèves qui imagineront en être les habitants. Utilisez ce lieu-thème comme un lieu de vie pour localiser toutes les activités d'expression écrite et orale, toutes les activités de communication générale et spécialisée. Vous obtenez ainsi une « simulation » méthodologique élaborée au BELC et pratiquée par les apprenants de français, en formation initiale ou en formation continue. (F. Mourlhon-Dallies, 2008, p.284]. Toute simulation s'inscrit dans un lieu thème. Pour les étudiants en droit, par exemple, la simulation s'organise sur un décor des lieux proches de ceux dans lesquels ils vont travailler à la sortie de la faculté. Ainsi, la simulation permettra à l'apprenant de se « mettre dans la peau » d'un personnage pour mieux comprendre une situation et les réactions possibles face à cette situation 
professionnelle. Cette méthode développe les attitudes favorisant la communication et la création.

La simulation permettra, ainsi, aux étudiants d enter dans «la peau d'un acteur juridique » et d'utiliser correctement dans un contexte professionnel la terminologie de spécialité. Les principes de la communication ne sont pas que ceux de la communication verbale, ils incluent aussi les gestes, l'image personnelle qu'on donne aux autres, les emphases dans le discours... etc.

Une bonne simulation juridique permettra de travailler les 4 compétences linguistiques:

- Compréhension orale et compréhension écrite,

- Expression orale et expression écrite.

Afin de se rapprocher du discours juridique le plus possible et de la para-communication en situation juridique tout en conservant l'intérêt des étudiants, on a décidé de simuler des procédures juridiques imaginées par les étudiants en droit après l'étude d'un cas.

Exemple d'une séquence de simulation a un cours de Français juridique.

Cas à défendre : Avocate et musicienne de rue

Bergerac (AFP) - Une avocate de Bergerac qui joue régulièrement de l'accordéon avec son ami violoniste sur les marchés de Dordogne suscite les foudres de ses pairs qui l'ont convoquée en conseil de discipline en l'accusant de manquer à son "devoir de dignité".

Me Valérie Faure, 42 ans dont sept de plaidoiries, devra s'expliquer le 7 octobre devant le conseil de discipline des avocats du barreau de Bergerac. La sanction peut aller du simple avertissement à la radiation, en passant par le blâme et la suspension temporaire. Pour le bâtonnier, Me Christian Tomme, le pire est que sa jeune consœur se livre publiquement à la mendicité. "On l'a vue sur des marchés et sur des foires, un chapeau posé devant elle pour recevoir des pièces", s'indigne-t-il. Pour lui, "ces agissements peuvent constituer une infraction à la déontologie et au devoir de dignité des avocats". De son côté, Me Faure assure que "tous les musiciens de rue posent un chapeau ou un étui devant eux, parce que c'est une tradition du métier". Néanmoins, il ne s'agit pas de mendicité parce que "les musiciens de rues ne sont pas des mendiants mais des artistes qui se produisent par envie déjouer, non pour demander la charité", plaidet-elle.

Pour elle, l'action engagée par le barreau de Bergerac relève de l'atteinte grave à la liberté individuelle et à la liberté d'expression, parce "qu'aucun texte ne dit que les avocats ne peuvent être musiciens des rues". "Il n'y a pas d'indignité de la profession, il y a une éthique à respecter, des valeurs de moralité et d'humanité, je ne vois pas en quoi je les enfreins quand je prends mon accordéon", affirme-t-elle. "J'aime mon métier, la musique classique est ma passion, jouer avec son compagnon sur les marchés ma joie de vivre". Et elle ne voit pas "pourquoi il faudrait choisir".

Models d'activités à proposer :

a. Organisez un débat sur le cas à défendre.

b. Préparez une plaidoirie pour défendre la jeune avocate.

c. Préparer la plaidoirie pour l'avocat d'accusation.

Face à un groupe d'étudiants en français juridique, le scénario commencera par:

- la description de la profession d'avocat;

- la description de la compétence d'un Tribunal;

- la personnalisation de la réponse;

- une conclusion.

Consignes pour les enseignants: Les élèves sont divisés en trois groupes, selon leur niveau :

Le premier groupe comporte les étudiants ayant un très bon niveau de la langue et qui peuvent être caractérisés comme inventifs et capables de prendre une idée et la développer. Le deuxième groupe comporte les étudiants à un niveau aussi bon de français, mais qui ont une réaction plus modérée face à une situation compétitive, mais sont reconnu comme« exécutifs ». Le dernier groupe se composé d'un groupe plus calmes et sur le plan d'action et sur le plan de réflexion.

L'enseignant proposera des activités qui correspondent à la communication orale et à la para-communication juridique. Lors de cette séance, on distribua les rôles et chacun simula une procédure juridique. Les manières de corriger l'oral peuvent être différentes:

En cas d'une erreur récurrente, on commence par arrêter la simulation pour corriger. Si l'erreur est causée par un manque de connaissances, de lexique, ou une mauvaise prononciation, le terme est écrit au tableau. S'il y a des erreurs de para-communication, on les recense et on les commente à la fin du cours.

Donc, une simulation peut être utilisée pour voir comment les étudiants réagiraient devant un certain événement ou face à certaines contraintes. Dans certaines simulations l'expérimentation est encouragée. 
Les principales étapes à suivre sont les suivantes:

- La simulation nécessite une grande clarté: Que veut-on illustrer par cette simulation?

- La fonction de chaque groupe ou de chaque personne participant à la simulation doit être identifiée.

- Aider les étudiants à utiliser leurs compétences et leurs connaissances pour traiter des problèmes qu'ils affrontent réellement.

Utilisation de la vidéo. Elles peuvent être utilisées comme introduction, comme stimulant pour une discussion ou comme résumé de points clés.

L'utilisation de vidéos nécessite une préparation préalable. Les enseignants devront:

- Choisir des documents culturellement appropriés;

- Savoir comment ils vont utiliser la vidéo et quels sont les questions/problèmes qu'ils désirent que les participants examinent:

- S'assurer que tous les participants peuvent voir l'écran et entendre le son.

Exemples de situations où l'utilisation de vidéos peut être efficace

Exemples de cette technique

Fiche Pedagogique Au Document Video

Niveau intermédiaire / B1

Document : Le droit

Objectifs communicatifs :

1. Comprendre globalement puis en détail un reportage.

2. Interpréter des informations.

3. Retrouver les liens entre différentes informations.

4. Imaginer puis faire un sondage.

Objectifs linguistiques :

1. Revoir les comparatifs.

2. Exprimer la cause et la conséquence.

Objectif interculturel :

1. S'interroger sur les attentes du monde professionnel.

Suggestions d'activités pour la classe

Suggestion de mise en route :

En petits groupes. Discutez sur les droits de l'homme.

Thème pour la discussion en groupe : » La loi-source principale de notre droit ».

Activité 1 : Préciser le rôle de la loi dans une société civile.

Suggestion pour l'activité 1 :

Montrer le reportage avec le son, mais sans les sous-titres jusqu'au début de l'intervention de DENIS SALASmagistrat professionnel.

Faites l'activité 2.

Demander aux apprenants de décrire la situation le plus précisément possible en s'aidant aussi du

Commentaire proposé par M. Salas. Faire une mise en commun à l'oral, inciter les apprenants à donner leur ressenti face aux premières images du reportage.

II ne s'agit pas de comprendre en détail les informations, mais d'avoir une vision globale de la situation.

Suggestion pour l'activité 3 : Séquence 3.

Apres avoir montrer la séquence 2 aux étudiants, montrer le reportage en entier avec le son et toujours sans les sous-titres.

Activité 3 :

Trouvez la différence entre :

Loi. ..... Coutume

Application :

Une femme mariée porte le nom de son mari. II s'agit d'une loi ou une coutume?

Activité 4. À deux.

Montrer à nouveau le reportage si nécessaire. Faire une mise en commun à l'oral et préparez la discussion en 2 : changer ou non le nom dans le mariage. Connaissez-vous les traditions dans d'autres pays ?

Cette activité permet de vérifier la compréhension détaillée d'informations contenues à la fois dans les images et dans le commentaire. 
Activité 5 « La langue du droit »: droit » :

Retenez et trouvez l'équivalent en roumain des mots et expressions proposés par la séquence »La langue du

- droit objectif

- droit objectif

- conseil constitutionnel

- la promulgation

Activité 6.

Demander aux apprenants de lire les phrases proposées par la séquence » Le mot dans l'histoire » :

Activité 7. Faire expliquer l'idée de l'art.6 : »La loi est l'expression de la volonté générale ».

Montrer le reportage sans les sous-titres. Faites-les parler du contenu de l'art. 6 .

Faites l'activité 8.

Montrer à nouveau le reportage pour permettre aux apprenants de justifier leurs réponses.

Activité 9. Répondez aux questions : séquence min.12.29

1. Quel est le plus ancien texte de loi ?

2. Quel est que le Code de Mourabi ?

3. Quelle a été la première loi roumaine ?

4. De quel siècle datte-elle?

5. Quelle a été la première loi barbare ?

6. En quoi consiste la première ordonnance de Louis XIV, roi de France?

Activité 9 . Faire une mise en commun à l'oral.

Cette activité demande aux apprenants de repérer certaines informations du reportage, d'en comprendre les détails ou de les interpréter.

Suggestion pour l'activité $4: 12.29$

Montrer le reportage avec les sous-titres.

Activité 10. En groupe. Donnez votre avis sur l'affaire du viol propose dans la séquence. Rédigez un petit commentaire sur le sujet : Viol-attente physique ou morale?

Activité 10. » Le bon ordre ».

Passer auprès des apprenants pour les aider et corriger leur production. Faire une mise en commun à l'oral et inviter chaque groupe à écrire au tableau quelques phrases.

Cette activité permet de relier les différentes informations vues dans les activités précédentes. II s'agit de vérifier la bonne compréhension des informations par les apprenants.

Suggestion pour l'activité 10 : Apres avoir visionné la séquence avec son, fermez le son et proposez aux étudiants de commentez les schémas proposées.

Activité 11. Former des petits groupes de 5 ou 6 apprenants. Leur préciser le travail attendu : ils peuvent poser des questions diverses sur le document.

Cette activité permet aux apprenants de s'entraîner à communiquer de façon réelle, si possible à l'extérieur de la classe et à s'exprimer en continu en langue étrangère. C'est aussi un moyen de les amener à s'interroger sur les différentes façons de voir le monde professionnel.

Activité 12. Regardez le reportage jusqu'au début de l'intervention de Denis Salas. Répondez aux

questions suivantes.

1. A partir duquel moment une loi est-elle applicable?

2. Quelles sont les étapes de l'application de la loi?

3. Quel est le droit le plus appliqué par les tribunaux?

\section{Conclusions}

En matière de méthodes pédagogiques, il faut savoir qu'il n'existe pas de bonne ou de mauvaise pédagogie en soi. Une méthode doit d'abord être adaptée à l'objectif fixé et au contenu de l'enseignement : c'est ce qu'on appelle la règle de contingence ou parfois le principe de contingence ce qui signifie qu'en la matière, il n'y a pas de règle absolue qui s'applique automatiquement dans tel ou tel cas d'enseignement ou de projet pédagogique. Simplement, certaines méthodes sont a priori mieux adaptées à telle intervention pédagogique; à l'intervenant de confronter a priori à la réalité de son intervention. 


\section{Références bibliographiques}

Ardouin T. (2003). Ingénierie de formation pour l'entreprise : analyser, concevoir, réaliser, évaluer. Paris, Dunod, p.274. Mourion-Dallies F, Didier, Enseigner une langue à des fins professionnelles Paris 2008, p.243.

Lera P. Les langues spécialisées, Paris, pub, 1995.

Sources internet : http://www.easydroit.fr/

www.marges-linguistiques.com 